\section{ORIGINAL RESEARCH}

K.B. Walhovd

A.M. Fjell

J. Brewer

L.K. McEvoy

C. Fennema-Notestine

D.J. Hagler, Jr

R.G. Jennings

D. Karow

A.M. Dale, and the Alzheimer's

Disease

Neuroimaging Initiative

\title{
Combining MR Imaging, Positron-Emission Tomography, and CSF Biomarkers in the Diagnosis and Prognosis of Alzheimer Disease
}

\begin{abstract}
BACKGROUND AND PURPOSE: Different biomarkers for AD may potentially be complementary in diagnosis and prognosis of AD. Our aim was to combine MR imaging, FDG-PET, and CSF biomarkers in the diagnostic classification and 2-year prognosis of $\mathrm{MCl}$ and $\mathrm{AD}$, by examining the following: 1) which measures are most sensitive to diagnostic status, 2) to what extent the methods provide unique information in diagnostic classification, and 3) which measures are most predictive of clinical decline.

MATERIALS AND METHODS: ADNI baseline MR imaging, FDG-PET, and CSF data from 42 controls, 73 patients with $\mathrm{MCl}$, and 38 patients with $\mathrm{AD}$; and 2-year clinical follow-up data for 36 controls, 51 patients with $\mathrm{MCl}$, and 25 patients with $\mathrm{AD}$ were analyzed. The hippocampus and entorhinal, parahippocampal, retrosplenial, precuneus, inferior parietal, supramarginal, middle temporal, lateral, and medial orbitofrontal cortices were used as regions of interest. CSF variables included A $\beta 42, t$-tau, $\mathrm{p}$-tau, and ratios of t-tau/A $\beta 42$ and $\mathrm{p}$-tau/A $\beta 42$. Regression analyses were performed to determine the sensitivity of measures to diagnostic status as well as 2-year change in CDR-SB, MMSE, and delayed logical memory in $\mathrm{MCl}$.
\end{abstract}

RESULTS: Hippocampal volume, retrosplenial thickness, and t-tau/A $\beta 42$ uniquely predicted diagnostic group. Change in CDR-SB was best predicted by retrosplenial thickness; MMSE, by retrosplenial metabolism and thickness; and delayed logical memory, by hippocampal volume.

CONCLUSIONS: All biomarkers were sensitive to the diagnostic group. Combining MR imaging morphometry and CSF biomarkers improved diagnostic classification (controls versus AD). MR imaging morphometry and PET were largely overlapping in value for discrimination. Baseline MR imaging and PET measures were more predictive of clinical change in $\mathrm{MCl}$ than were CSF measures.
ABBREVIATIONS: $\mathrm{A} \beta 42=\beta$ amyloid $1-42 ; \mathrm{AD}=$ Alzheimer disease; $\mathrm{ADNI}=$ Alzheimer's Disease Neuroimaging Initiative; $A \cup C=$ area under the curve; $B=B$ coefficient for each predictor in the regression equation; CDR-SB = Clinical Dementia Rating sum of boxes; Corr. Class. = correlation classification; $\mathrm{CSHC}=$ Center for the Study of Human Cognition; FDA = US Food and Drug Administration; 18F-FDG $=\left[{ }^{18} \mathrm{~F}\right]$ fluorodeoxyglucose; FDG-PET = fluorodeoxyglucose-positronemission tomography; inf. = inferior; lat. = lateral; LM-del = delayed Logical Memory from the Wechsler Memory Scale Logical Memory II; $\mathrm{M}=$ mean; $\mathrm{MCl}=$ mild cognitive impairment; med orb. front. = medial orbital frontal; mid = middle; MMSE = Mini-Mental State Examination; $\mathrm{MRI}=$ MR imaging; $\mathrm{NIH}=$ National Institutes of Health; $\mathrm{NC}=$ healthy control; orb. front. = orbital frontal; p-tau $=$ phosphorylated tau protein 181; parahippoc. $=$ parahippocampus; $\mathrm{PET}=$ positron-emission tomography; $\mathrm{t}$-tau $=$ tau protein; $\mathrm{ROC}=$ receiver operating characteristics
$\mathbf{M}$ ultiple biomarkers have proved sensitive to $\mathrm{AD}$ and $\mathrm{MCI}$, a potential prodromal stage of $\mathrm{AD}$. These include patterns of regional cerebral atrophy and hypometabolism detected by MR imaging and FDG-PET ${ }^{1}$ and quantification of specific proteins in the CSF, including the tau protein and

Received May 20, 2009; accepted after revision July 1.

From Department of Psychology (K.B.W., A.M.F.), CSHC, University of Oslo, Oslo, Norway; Department of Neuropsychology (K.B.W., A.M.F.), Ulleval University Hospital, Oslo, Norway; Departments of Neuroscience (J.B., A.M.D.), Radiology (J.B., L.K.M., C.F.-N., D.J.H., R.G.J., D.K., A.M.D.), and Psychiatry (C.F.-N.), University of California, San Diego, La Jolla, California.

This work was supported by grants from the National Center for Research Resources (U24 RR021382-CFN, AMD, DJH) and the National Institute on Aging (R01AG22381-CFN, LKM, AMD; K01AG029218- LKM). The work of K.B. Walhovd and A.M. Fjell was supported by the Norwegian Research Council. Data collection and sharing for this project were funded by the ADNI (Principal Investigator, Michael Weiner; NIH grant U01 AG024904). The ADNI is funded by the National Institute on Aging, National Institute of Biomedical Imaging and Bioengineering, and through generous contributions from the following companies and organizations: Pfizer, Wyeth Research, Bristol-Myers Squibb, Eli Lilly and Company, GlaxoSmithKline, Merck \& Co, AstraZeneca, Novartis Pharmaceuticals Corporation, the Alzheimer's Association, Eisai Global Clinical Development, Elan Corporation, Forest Laboratories, and the Institute for the Study of Aging with participation from the FDA. Industry partnerships are coordinated through the Foundation for the $\mathrm{NIH}$. The grantee organization
$\mathrm{A} \beta 42 .{ }^{2}$ Tau is associated with axonal microtubules and is the main structural element of neurofibrils in AD. High CSF tau levels probably reflect axonal degeneration. ${ }^{3} \mathrm{~A} \beta 42$ is derived from cleavage of amyloid precursor protein, and CSF A $\beta 42$ levels are lowered early in $\mathrm{AD}$, possibly due to sequestering of

is the Northern California Institute for Research and Education, and the study is coordinated by the Alzheimer's Disease Cooperative Study at the University of California, San Diego. ADNI data are disseminated by the Laboratory of Neuro Imaging at the University of California, Los Angeles.

Data used in the preparation of this article were obtained from the ADNI data base (www.loni.ucla.edu/ADNI). As such, the investigators within the ADNI contributed to the design and implementation of $\mathrm{ADNI}$ and/or provided data but did not participate in the analysis or writing of this article. A complete listing of ADNI investigators is available at: http://www.Ioni.ucla.edu/ADNI/Data/ADNI_Authorship_List.pdf.

Please address correspondence to Kristine B. Walhovd, MD, Department of Psychology, CSHC, University of Oslo, POB 1094 Blindern, 0317 Oslo, Norway; e-mail: k.b.walhovd@ psykologi.uio.no

Indicates open access to non-subscribers at www.ajnr.org

Indicates article with supplemental on-line tables.

DOI 10.3174/ajnr.A1809 
Table 1: Demographic characteristics of the 3 subsamples ${ }^{a}$

\begin{tabular}{|c|c|c|c|c|c|c|c|c|c|}
\hline & \multicolumn{3}{|c|}{$\mathrm{NC}(n=42 ; 16 \mathrm{~F} / 26 \mathrm{M})$} & \multicolumn{3}{|c|}{$\mathrm{MCI}(n=73,25 \mathrm{~F} / 48 \mathrm{M})$} & \multicolumn{3}{|c|}{$\mathrm{AD}(n=38,16 \mathrm{~F} / 22 \mathrm{M})$} \\
\hline & $\mathrm{M}$ & SD & Range & $\mathrm{M}$ & SD & Range & $\mathrm{M}$ & SD & Range \\
\hline Age & 75.5 & (5.4) & $62.2-84.7$ & 74.5 & $(7.0)$ & $55.5-88.9$ & 76.2 & (7.5) & $58.8-88.1$ \\
\hline Education & 16.0 & (3.2) & 8-20 & 16.0 & $(2.9)$ & 8-20 & 14.3 & (3.6) & 4-20 \\
\hline MMSE & 29.1 & (1.0) & $26-30$ & 27.0 & $(1.7)$ & $24-30$ & 23.8 & (2.0) & $20-26$ \\
\hline MMSE_c & -0.2 & (1.6) & $-4-3$ & -1.3 & (2.8) & $-13-4$ & -5.2 & (5.8) & $-22-4$ \\
\hline CDR & 0.0 & $(0.0)$ & 0-0 & 0.5 & $(0.0)$ & $0.5-0.5$ & 0.8 & $(0.3)$ & 0.5-1.0 \\
\hline CDR_c & 0.2 & $(0.7)$ & $-0.5-3.5$ & 1.2 & $(1.6)$ & $-1.5-4.5$ & 4.0 & (3.1) & 0-11 \\
\hline LM-del & 12.0 & (3.6) & $6-22$ & 4.1 & (2.7) & $0-8$ & 1.1 & (2.0) & $0-8$ \\
\hline LM-del_c & 1.2 & (4.1) & $-10-8$ & 0 & (3.3) & $-6-10$ & -0.7 & (1.1) & $-4-1$ \\
\hline
\end{tabular}

a The numbers refer to baseline data, with the exception of MMSE c, CDR c, and LM-del c, which refer to change across 2 years (baseline score subtracted from score at 2-year follow-up). MMSE and LM-del change scores were available for $36 \mathrm{NC}, 51 \mathrm{MCl}$, and $25 \mathrm{AD}$ subjects. CDR-SB change scores were available for $34 \mathrm{NC}, 49 \mathrm{MCl}$, and $25 \mathrm{AD}$.

A $\beta 42$ in neuritic plaques. ${ }^{4}$ A full spectrum of imaging and CSF analysis methods is seldom used; thus, knowledge is limited on how they may best be combined. The ADNI, a large multisite study, was launched to enable analyses of combinations of different candidate biomarkers for AD.

Recent findings indicate that MR imaging can be used to quantify regional atrophy in $\mathrm{MCI}$, distinguishing early and later preclinical stages of $\mathrm{AD},{ }^{5}$ and such measures are predictive of clinical decline across 1 year. $^{6-8}$ A pattern of parietotemporal metabolic reductions in $\mathrm{MCI}$ and $\mathrm{AD}$ and frontal metabolic reductions later in the disease has been established through the last decades of research ${ }^{1,9,10}$ and has recently been confirmed in ADNI PET data. ${ }^{11}$ The relative sensitivity of FDG-PET and MR imaging morphometry to AD-related changes is, however, not well established. It has been assumed that metabolic changes associated with neocortical dysfunction may be detectable by FDG-PET before atrophy appears. Consistent with this assumption, De Santi et $\mathrm{al}^{12}$ reported that metabolism reductions exceeded volume losses in MCI, and Mosconi et $\mathrm{al}^{13}$ found the same in presymptomatic early-onset familial AD. However, Jagust et $\mathrm{al}^{14}$ found that cingulate hypometabolism was a significant risk factor in addition to MR imaging measures of hippocampal atrophy, but the latter was a more statistically robust risk factor in a group of cognitively impaired but not demented elderly. ${ }^{15}$

Different brain characteristics relevant for the understanding of MCI and AD may be captured by FDG-PET and MR imaging morphometry. For instance, a report based on ADNI data has indicated that FDG-PET and MR imaging measures may be complementary and differentially sensitive to memory in health and disease, with metabolism being the stronger predictor in healthy controls and morphometry most related to memory function in $\mathrm{AD} .{ }^{16}$ As for CSF-MR imaging relations, recent reports ${ }^{17-22}$ indicate that cerebral anatomic differences are related to tau and $\mathrm{A} \beta 42$ and behavioral cognitive measures in AD and MCI. However, MR imaging and CSF biomarkers have not simultaneously been related and compared with information obtained by FDG-PET. It is important to test the specific sensitivity of all biomarkers simultaneously to be able to optimize the combination of measures in diagnosis and prognosis. We investigated the following: 1) which methods are the most sensitive to established AD-related pathology, 2) to what extent the methods provide unique-versus-overlapping information, and 3) which methods are the most predictive of clinical decline across 2 years.

\section{Materials and Methods}

The raw data used in the preparation of this article were obtained from the ADNI data base (www.loni.ucla.edu/ADNI). ADNI was launched in 2003 by the National Institute on Aging, the National Institute of Biomedical Imaging and Bioengineering, the FDA, private pharmaceutical companies, and nonprofit organizations. The primary goal of ADNI has been to test whether serial MR imaging, PET, other biologic markers, and clinical and neuropsychological assessment can be combined to measure the progression of MCI and early AD. The Principal Investigator of this initiative is Michael W. Weiner, of the Veterans Administration Medical Center and University of California-San Francisco. There are many coinvestigators, and subjects have been recruited from $>50$ sites across the United States and Canada. The ADNI has recruited 229 healthy elderly subjects, 398 patients with MCI, and 192 patients with AD to participate and be followed for 2-3 years. For up-to-date information, see www.adniinfo.org.

\section{Sample}

ADNI eligibility criteria are described at http://www.adni-info.org/ index.php?option $=$ com_content $\&$ task $=$ view\&id $=9 \&$ Itemid $=43$. Briefly, participants were 55-90 years of age, had an informant providing an independent evaluation of functioning, and spoke English or Spanish. Subjects were willing and able to undergo test procedures, including neuroimaging and longitudinal follow-up, and all gave informed consent. Specific psychoactive medications were excluded. General inclusion/exclusion criteria of the ADNI study are as follows: 1) healthy subjects: $\mathrm{MMSE}^{23}$ scores between 24 and 30 inclusive (no person enrolled as an NC in the present sample had an MMSE score below 26); CDR of 0, nondepressed, non-MCI, and nondemented; 2) subjects with MCI: MMSE scores between 24 and 30 inclusive (exceptions made on a case-by-case basis, but no such exceptional cases were enrolled as patients with MCI in the present sample), a memory complaint, objective memory loss measured by Wechsler Memory Scale Logical Memory II, ${ }^{24}$ CDR of 0.5, absence of significant levels of impairment in other cognitive domains, essentially preserved activities of daily living, and an absence of dementia; and 3) mild AD: MMSE scores between 20 and 26 inclusive (exceptions made on a case-by-case basis), CDR of 0.5 or 1.0, and met the National Institute of Neurological and Communicative Disorders and Stroke and the Alzheimer's Disease and Related Disorders Association criteria for probable AD. ${ }^{25}$ Only ADNI subjects for whom adequate processed and quality checked MR imaging, FDG-PET, and CSF baseline data were available were included. This yielded a total of 153 participants. Demographics are shown in Table 1. 


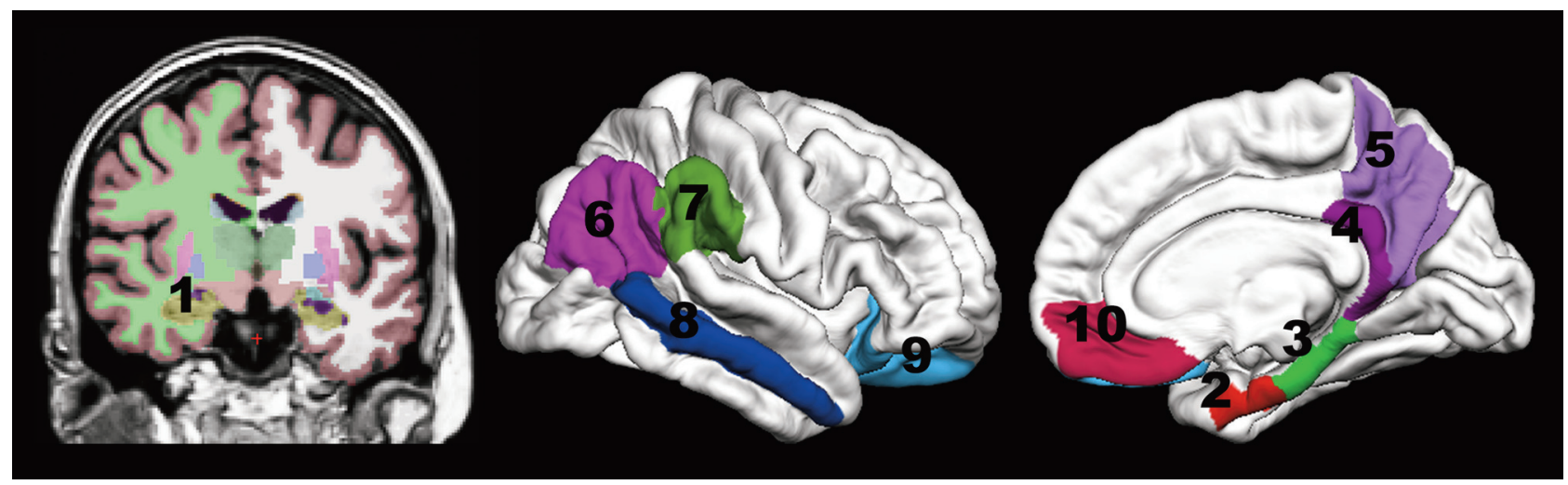

Fig 1. The regions of interest used are the following: 1) hippocampus and 2) entorhinal, 3) parahippocampal, 4) retrosplenial, 5) precuneus, 6) inferior parietal, 7) supramarginal, 8) middle temporal, 9) lateral orbitofrontal, and 10) medial orbitofrontal cortices.

\section{Standard Protocol Approvals, Registrations, and Patient Consents}

The protocol was approved by the institutional review boards of participating sites. Written informed consent was obtained from all subjects or from guardians of patients.

\section{MR Imaging Acquisition and Analysis}

All scans used here were from 1.5T scanners. Data were collected across a variety of scanners with protocols individualized for each scanner, as defined at http://www.loni.ucla.edu/ADNI/Research/ Cores/index.shtml, and processed as described elsewhere. ${ }^{5,16}$ Briefly, raw DICOM MR imaging scans (including 2 T1-weighted volumes per case) were downloaded from the ADNI site (http://www.loni. ucla.edu/ADNI/Data/index.shtml), reviewed for quality, automatically corrected for spatial distortion due to gradient nonlinearity ${ }^{26}$ and $\mathrm{B}_{1}$ field inhomogeneity, ${ }^{27}$ registered, and averaged to improve signal intensity-to-noise ratios. Scans were segmented as described by Fischl et al, ${ }^{28}$ yielding volumetric data for the hippocampal formation (consisting of the dentate gyrus, Cornu Ammonis fields, subiculum/parasubiculum, and the fimbria ${ }^{29}$ ).

The procedure ${ }^{28,30}$ uses a probabilistic atlas and applies a Bayesian classification rule to assign a neuroanatomic label to each voxel. The cortical surface was reconstructed to measure thickness at each surface point by using a semiautomated approach described elsewhere. ${ }^{31-36}$ Thickness measurements were obtained by reconstructing representations of the gray/white matter boundary ${ }^{31,32}$ and the pial surface and then calculating the distance between those surfaces at each point across the cortical mantle. The measurement technique used here has been validated via histologic ${ }^{37}$ and manual measurements. ${ }^{38}$ The entire cortical surface was parcellated into numerous cortical areas. ${ }^{30,39}$ To limit multiple comparisons, we selected candidate regions of interest on the basis of previous MR imaging and PET findings ${ }^{1,5,7,11,16,40-44}$ indicating sensitivity to $\mathrm{AD}$-related pathology: the hippocampi, and the entorhinal, parahippocampal, retrosplenial, precuneus, inferior parietal, supramarginal, middle temporal, and lateral and medial orbitofrontal gyri.

In the parcellation method used here, ${ }^{39}$ the entire cingulate cortex was defined and divided into 4 separate regions, including the rostral and caudal anterior cingulate, the posterior cingulate, and the isthmus cingulate, the latter referred to here as the retrosplenial cortex for consistency with other published studies. ${ }^{5,16,40}$ The retrosplenial region may also be referred to as the isthmus of the cingulate or caudal posterior cingulate area in other contexts. For a depiction of the exact regions of interest used, see Fig 1.

\section{FDG-PET Acquisition and Analysis}

Subjects were scanned after a 4 -hour fast (water only). Plasma glucose had to be $\leq 180 \mathrm{mg} / \mathrm{dL}$ for FDG to be injected. An intravenous catheter was placed in $1 \mathrm{arm}$ for injection of 18F-FDG. Imaging began at 30 minutes postinjection, and the scan was acquired as six 5-minute frames. For each subject, FDG-PET frames were averaged and registered to the corresponding distortion-corrected and intensity-normalized MR imaging volume. PET activity for each subject was sampled onto their reconstructed cortical surface, averaged within each region of interest, and normalized to activity within the pons. ${ }^{45}$

\section{CSF Acquisition and Analysis}

CSF samples obtained by lumbar puncture were examined for $\mathrm{t}$-tau, p-tau, and $\mathrm{A} \beta 42$ by using an immunoassay method. ${ }^{46}$ The measurements were performed by L. Shaw and J. Trojanowski of the ADNI Biomarker Core at the University of Pennsylvania School of Medicine. We analyzed the following CSF biomarkers for the present article: A $\beta 42(202 \pm 56,159 \pm 51,136 \pm 39 \mathrm{pg} / \mathrm{mL}$ for NC, MCI, and AD, respectively), t-tau $(68 \pm 28,100 \pm 65,125 \pm 67 \mathrm{pg} / \mathrm{mL}$ for NC, MCI, and $\mathrm{AD}$, respectively), and p-tau $(26 \pm 17,36 \pm 19,45 \pm 23$ for NC, $\mathrm{MCI}$, and $\mathrm{AD}$, respectively). The ratios of tau and $\mathrm{A} \beta 42$ (tau/A $\beta 42$; $0.37 \pm 0.21,0.74 \pm 0.67,0.98 \pm 0.56$ for $\mathrm{NC}, \mathrm{MCI}$, and $\mathrm{AD}$, respectively) and the p-tau $\mathrm{A} \beta 42$ ratio (p-tau/A $\beta 42 ; 0.16 \pm 0.16,0.26 \pm$ $0.19,0.36 \pm 0.22$ for $\mathrm{NC}, \mathrm{MCI}$, and $\mathrm{AD}$, respectively) were also included. A 1-way analysis of variance on the residual CSF values after age and sex were regressed out showed significant $(P<.001)$ main effects of group on all variables. Post hoc tests controlling for multiple comparisons showed significant $(P<.05)$ differences between NC and $\mathrm{MCI}, \mathrm{NC}$ and $\mathrm{AD}$, and $\mathrm{MCI}$ and $\mathrm{AD}$, with a few exceptions where trends $(P<.10)$ were observed (differences in $\mathrm{t}$-tau between $\mathrm{MCI}$ and $\mathrm{AD}$, p-tau between NC and MCI, and t-tau/A $\beta 42$ between MCI and $\mathrm{AD})$.

\section{Clinical and Cognitive Measures}

Change scores were calculated by subtracting baseline scores from scores obtained at the 2-year follow-up. In addition to CDR-SB ${ }^{47}$ and MMSE, ${ }^{23}$ delayed recall on the Wechsler Memory Scale-Revised ${ }^{48}$ was included. This test requires the subject to recall a story read by the examiner after a 30- to 40-minute delay and is sensitive to the episodic memory deficits in MCI.

\section{Statistics}

A repeated-measures general linear model with the 10 regions of interest $\times$ hemisphere (left, right $) \times$ diagnostic group $(\mathrm{NC}, \mathrm{MCI}, \mathrm{AD})$ 


\begin{tabular}{|c|c|c|c|c|c|c|c|}
\hline Method & Step & Measure & $\mathrm{B}$ & $P$ & Odds Ratio & $\%$ Corr. Class. & $R^{2 \mathrm{a}}$ \\
\hline \multirow[t]{3}{*}{$\mathrm{MRI}$} & 1 & Hippocampus & -2.306 & .000 & .100 & $\begin{array}{l}\text { NC:83.3 } \\
\text { AD:81.6 } \\
\text { All: } 82.5\end{array}$ & .601 \\
\hline & 2 & $\begin{array}{l}\text { Hippocampus } \\
\text { Retrosplenial cortex }\end{array}$ & $\begin{array}{l}-2.291 \\
-1.202\end{array}$ & $\begin{array}{l}.000 \\
.014\end{array}$ & $\begin{array}{l}.101 \\
.301\end{array}$ & $\begin{array}{l}\text { NC:88.1 } \\
\text { AD:78.9 } \\
\text { All: } 83.8\end{array}$ & .665 \\
\hline & 3 & $\begin{array}{l}\text { Hippocampus } \\
\text { Entorhinal cortex } \\
\text { Retrosplenial cortex }\end{array}$ & $\begin{array}{l}-1.581 \\
-1.314 \\
-1.230\end{array}$ & $\begin{array}{l}.011 \\
.026 \\
.024\end{array}$ & $\begin{array}{l}.206 \\
.269 \\
.292\end{array}$ & $\begin{array}{l}\text { NC: } 85.7 \\
\text { AD: } 84.2 \\
\text { All: } 85.0\end{array}$ & .714 \\
\hline \multirow[t]{3}{*}{ PET } & 1 & Entorhinal cortex & -1.627 & .000 & .197 & $\begin{array}{l}\text { NC:85.7 } \\
\text { AD:73.7 } \\
\text { All: } 80.0\end{array}$ & .461 \\
\hline & 2 & $\begin{array}{l}\text { Entorhinal cortex } \\
\text { Lateral orbitofrontal cortex }\end{array}$ & $\begin{array}{r}-2.142 \\
.675\end{array}$ & $\begin{array}{l}.000 \\
.048\end{array}$ & $\begin{array}{r}.117 \\
1.964\end{array}$ & $\begin{array}{l}\text { NC:81.0 } \\
\text { AD:76.3 } \\
\text { All:78.8 }\end{array}$ & .506 \\
\hline & 3 & $\begin{array}{l}\text { Entorhinal cortex } \\
\text { Retrosplenial cortex } \\
\text { Lateral orbitofrontal cortex }\end{array}$ & $\begin{array}{r}-2.094 \\
-1.866 \\
1.701\end{array}$ & $\begin{array}{l}.000 \\
.003 \\
.002\end{array}$ & $\begin{array}{r}.123 \\
.155 \\
5.481\end{array}$ & $\begin{array}{l}\text { NC: } 88.1 \\
\text { AD: } 76.3 \\
\text { All: } 82.5\end{array}$ & .620 \\
\hline CSF & 1 & $\mathrm{t}-\tau: \mathrm{A} \beta 42$ & 2.775 & .000 & 16.036 & $\begin{array}{l}\text { NC:85.7 } \\
\text { AD:76.3 } \\
\text { All: } 81.2\end{array}$ & .523 \\
\hline
\end{tabular}

${ }^{\text {a }}{ }^{2}$ is Nagelkerke $R^{2}$.

with age and sex as covariates showed no significant effect of hemisphere across regions of interest $(F[1,148]=1.530, P=.218)$ and no interaction of hemisphere $\times$ diagnostic group $(F[2,148]=0.847, P=$ $.431)$. Hence, values were averaged across hemispheres, effects of age and sex were regressed out, and the standardized residuals were used in the analyses. Correlation analyses with MR, FDG-PET, and CSF measures were run to assess their covariance. To select the measures yielding the most explained variance for each method, we entered the values in 3 separate logistic stepwise regressions by using MR, PET, and CSF measures respectively, predicting NC versus AD. The selected MR, PET, and CSF variables were then entered simultaneously in multimethod stepwise logistic regression analyses predicting NC versus $\mathrm{AD}$ and $\mathrm{NC}$ versus MCI. Next, the variables identified by the NC-versus-AD classification analysis were correlated with 2-year follow-up CDR-SB, MMSE, and delayed logical memory change scores in the MCI group and were entered as predictors in stepwise regression analyses with the respective behavioral change scores as the dependent variables.

\section{Results}

Correlation analyses in the MCI group for morphometry and metabolism for the 10 regions of interest and the 5 CSF variables showed no significant $(P<.05$, corrected for 10 regionof-interest comparisons) correlations among CSF variables and morphometry or metabolism in any region of interest, whereas moderate correlations were found between morphometric and metabolic measures for the hippocampus and entorhinal, retrosplenial, and inferior parietal regions (on-line Table).

Table 2 shows the results of the separate logistic stepwise regressions predicting $\mathrm{NC}$-versus-AD classification on the basis of MR imaging, FDG-PET, and CSF measures. Hippocampal volume and entorhinal and retrosplenial thickness, for MR imaging, were included in the final model, yielding an overall classification accuracy of $85.0 \%$, and approximately $71 \%$ explained variance (Nagelkerke $R^{2}$ ). Entorhinal, retrosplenial, and lateral orbitofrontal metabolism, for FDG-PET, were in- cluded in the final model, yielding an overall classification accuracy of $82.5 \%$ and approximately $62 \%$ explained variance. For CSF, the ratio of $\mathrm{t}$-tau/A $\beta 42$ was the single unique predictor, yielding an overall classification accuracy of $81.2 \%$, and approximately $52 \%$ explained variance. Thus, hippocampal volume; entorhinal and retrosplenial thickness; entorhinal, retrosplenial, and lateral orbitofrontal metabolism; and $\mathrm{t}$-tau/A $\beta 42$ ratio were entered in a logistic regression analysis to classify NC versus AD, and the results are shown in Table 3.

In the final model, hippocampal volume, retrosplenial thickness, and t-tau/A $\beta 42$-ratio were included as predictors, yielding an overall classification accuracy of $88.8 \%$ and approximately $78 \%$ explained variance. Figure 2 depicts the ROC curves for these variables when using 1 (hippocampal volume) versus a combination of 2 (hippocampal volume and $\mathrm{t}$-tau/A $\beta 42$-ratio) and all 3 variables (hippocampal volume, $\mathrm{t}$-tau/A $\beta 42$-ratio, and retrosplenial thickness) shown to be unique predictors of NC-versus-AD classification. Predicted values from logistic regressions were used for calculation of the ROC curves. Statistical comparisons of the AUCs of these classifiers were performed by using the method of Hanley and McNeil. ${ }^{49}$ This approach yielded a significant difference $(P<$. 05 ) between the AUCs using hippocampal volume alone versus using hippocampal volume and t-tau/A $\beta 42$ ratio in combination and hippocampal volume, $\mathrm{t}$-tau/A $\beta 42$ ratio, and retrosplenial thickness in combination. The difference of the AUCs using hippocampal volume and $t$-tau/A $\beta 42$ ratio versus hippocampal volume, t-tau/A $\beta 42$ ratio, and retrosplenial thickness in combination was clearly smaller and not significant $(P>$.05). Note however, that all meaningful differences in AUCs (eg, in terms of sensitivity versus specificity causing the curves to cross) may not necessarily be captured as statistically significant. The same set of predictor variables was entered in an analysis to predict diagnostic classification for $\mathrm{NC}$ and MCI, which revealed that hippocampal volume and t-tau/ $\mathrm{A} \beta 42$ ratio were unique predictors, yielding an overall classi- 


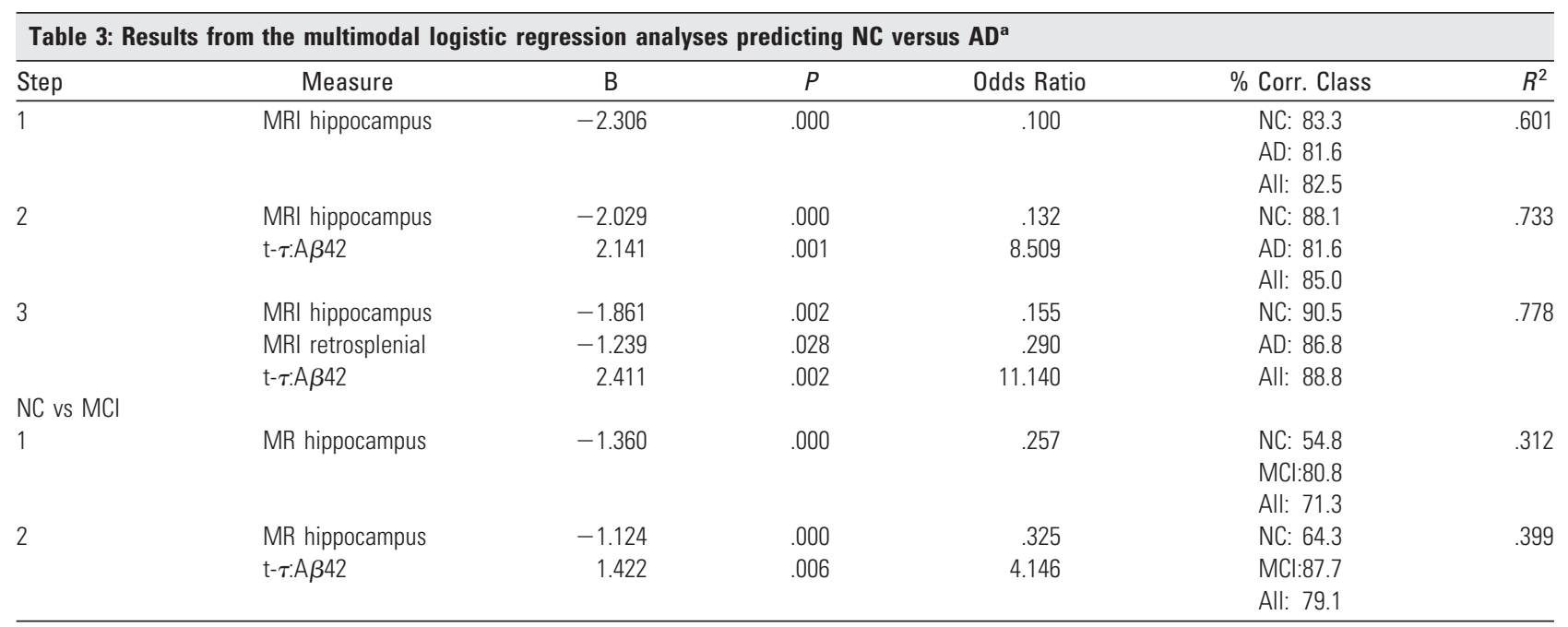

a The variables explaining unique variance within each method, as listed in Table 2, were included in the set of predictor variables, i.e. for MR: hippocampal volume, retrosplenial, and entorhinal thickness; for PET: entorhinal, retrosplenial, and lateral orbitofrontal metabolism; and for CSF: the ratio of T-tau to Abeta 42 . $R^{2}$ is Nagelkerke $R^{2}$.

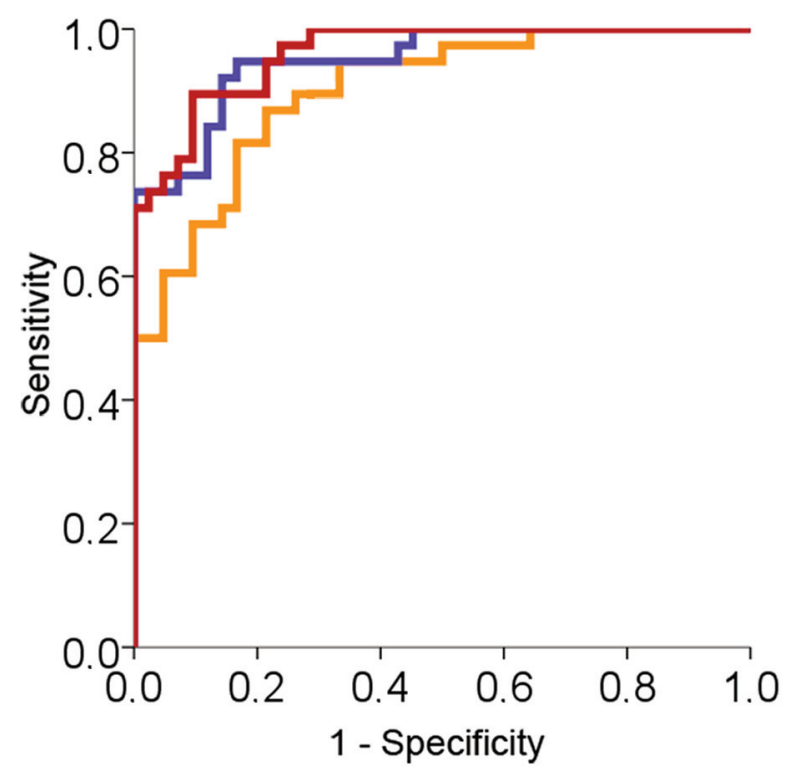

Fig 2. Comparison of ROC curves for using 1 versus a combination of 2 and all 3 variables shown to be unique predictors of NC-versus-AD classification. Yellow is the predicted probability based on hippocampal volume alone (AUC $=0.900, S E=0.033$ ). Blue is the predicted probability based on hippocampal volume and t-tau/A $\beta 42$ ratio (AUC $=0.950$, $\mathrm{SE}=0.022)$. Red is the predicted probability based on hippocampal volume, $\mathrm{t}$-tau/A $\beta 42$ ratio, and retrosplenial cortical thickness (AUC $=0.961, \mathrm{SE}=0.018$ ).

fication accuracy of $79.1 \%$ and approximately $40 \%$ explained variance.

Table 4 shows correlations for each variable included in the regression models and the cognitive change scores (CDR-SB, MMSE, delayed logical memory). In MCI, baseline retrosplenial thickness correlated with a 2-year change in CDR-SB and MMSE, where a thicker cortex was associated with less CDR-SB elevation and less MMSE reduction. Retrosplenial and entorhinal metabolism correlated negatively with MMSE change. Hippocampal volume correlated positively with delayed logical memory. There were no significant correlations with clinical change measures for t-tau/A $\beta 42$ in MCI. $T$ tests of the Fisher $z$-transformed correlation coefficients showed that the t-tau/A $\beta 42$ ratio correlated significantly lower $(P<$ $.05)$ with CDR-SB and MMSE change than did retrosplenial

\begin{tabular}{|c|c|c|c|}
\hline \multicolumn{4}{|c|}{$\begin{array}{l}\text { Table 4: Correlations between the variables included in the } \\
\text { regression models predicting NC/AD classification and the change } \\
\text { in CDR-SB }(n=49) \text { and MMSE }(n=51) \text { scores across } 2 \text { years in } \\
\text { the MCI group }\end{array}$} \\
\hline & CDR-SB Change & MMSE Change & LM-Del Change \\
\hline MRI hippocampus & -.29 & .29 & $.41^{\mathrm{b}}$ \\
\hline MRI entorhinal & -.17 & .23 & .34 \\
\hline MRI retrosplenial & $-.43^{b}$ & $.42^{\mathrm{b}}$ & .35 \\
\hline PET entorhinal & -.30 & $.38^{\mathrm{b}}$ & .28 \\
\hline PET retrosplenial & -.22 & $.47^{\mathrm{b}}$ & .11 \\
\hline PET lat. orbitofrontal & -.02 & .27 & -.05 \\
\hline $\mathrm{T}-\tau: \mathrm{A} \beta 42$ & .02 & .08 & -.23 \\
\hline
\end{tabular}

a The variables explaining the unique variance within each method, as listed in Table 2 . were included in the set of predictor variables (ie, for MR imaging, hippocampal volume and retrosplenial and entorhinal thickness; for PET, entorhinal, retrosplenial, and lateral orbitofrontal metabolism; and for CSF, the ratio of $\mathrm{t}-\tau: \mathrm{A} \beta 42$ )

b $P<.05$, corrected for 7 comparisons.

thickness and it correlated significantly lower with MMSE change than did entorhinal and retrosplenial metabolism. Lateral orbitofrontal metabolism also correlated significantly lower with change in CDR-SB and delayed logical memory than did retrosplenial thickness and hippocampal volume.

In the stepwise regression analysis predicting CDR-SB change, only retrosplenial cortical thickness was included as a unique predictor $(\mathrm{y}=1.231-0.731 \mathrm{x}, P=.002)$, explaining $18 \%$ of the variance. In predicting MMSE change, retrosplenial metabolism was included in the first step $(y=-1.193+$ $1.534 \mathrm{x}_{1}, P=.002$ for $\left.\mathrm{x}_{1}, R^{2}=0.22\right)$, and retrosplenial thickness was added in the second $\left(\mathrm{y}=-1.197+1.177 \mathrm{x}_{1}+0.776 \mathrm{x}_{2}\right.$, $P=.009$ for $\mathrm{x}_{1}$ and .042 for $\left.\mathrm{x}_{2}, R^{2}=0.29\right)$. Only hippocampal volume was included as a predictor of delayed logical memory change $\left(\mathrm{y}=0.240+1.669 \mathrm{x}_{1}, P=.003\right.$ for $\left.\mathrm{x}_{1}, R^{2}=0.17\right)$. The regression plots for CDR-SB and MMSE change predicted from retrosplenial thickness and metabolism and delayed logical memory predicted from hippocampal volume are shown in Fig 3. There was 1 outlier for the MMSE change score, with a 13-point decline. Without this outlier, only retrosplenial metabolism was included in the model for predicting MMSE change $\left(\mathrm{y}=-1.023+1.091 \mathrm{x}_{1}, P=.004\right.$ for $\left.\mathrm{x}_{1}, R^{2}=0.16\right)$, but a trend was observed for retrosplenial thickness $(P=$ $.079)$. 

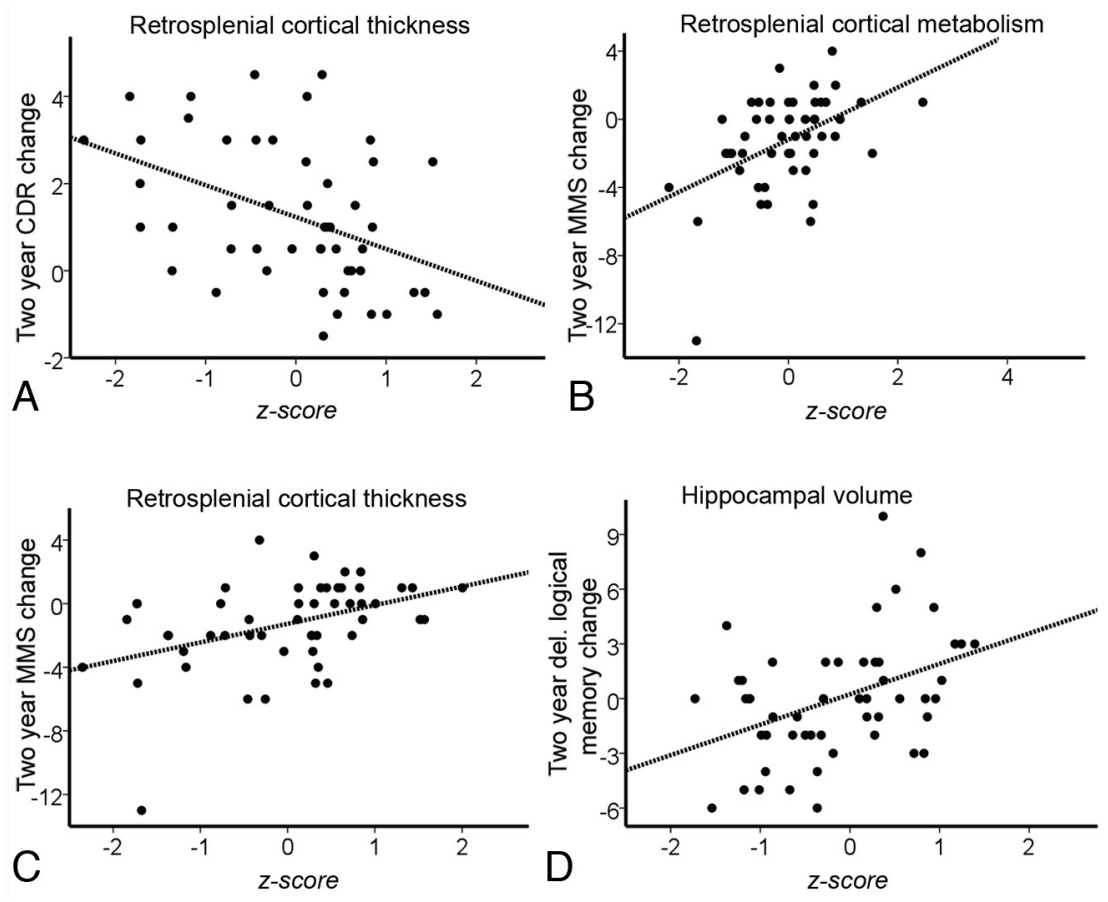

Fig 3. The regression plots for 2-year change in scores in the $\mathrm{MCl}$ group significantly $(P<.05)$ predicted from MR imaging morphometry and $\mathrm{PET}$ metabolism variables. $A$, CDR change predicted from retrosplenial cortical thickness. $B$ and $C$, MMSE change predicted from retrosplenial cortical metabolism $(B)$ and retrosplenial cortical thickness $(C)$. $D$, Delayed logical memory change predicted from hippocampal volume.

\section{Discussion}

Morphometry, metabolism, and CSF biomarkers were all sensitive to diagnostic status. The best classification accuracy of $\mathrm{NC}$ versus $\mathrm{AD}$ was obtained by MR imaging morphometry measures (hippocampal volume, entorhinal and retrosplenial cortical thickness). However, classification accuracies close to those obtained by MR imaging were also obtained by FDGPET (entorhinal, retrosplenial, and lateral orbitofrontal metabolism) and CSF measures (t-tau/A $\beta 42$-ratio). In the multimodal analysis, FDG-PET measures appeared to provide largely redundant information, whereas hippocampal volume, retrosplenial thickness, and the $\mathrm{t}$-tau/A $\beta 42$ ratio were unique predictors of diagnostic status. In particular, the inclusion of the CSF biomarker in addition to MR imaging hippocampal volume did result in a significant improvement in classification in terms of AUC. Thus, the combination of MR imaging morphometry and CSF biomarkers yielded the highest diagnostic classification accuracy. Contrary to this finding, in the prediction of clinical change during 2 years, FDG-PET and MR imaging morphometry were the best predictors. However, with the exception of retrosplenial metabolism and thickness in the prediction of change in MMSE scores, the 2 measures were largely redundant. Thus, it seems that the benefits of including both MR imaging morphometry and FDGPET are modest in predicting clinical decline in MCI.

Whereas CSF biomarkers added to the diagnostic accuracy at baseline, they did not predict 2-year clinical decline in the current MCI group. This finding may be somewhat surprising because previous studies have found decreased CSF A $\beta 42$ and/or tau or tau/A $\beta 42$ levels to be predictive of future dementia in patients with MCI. ${ }^{2}$ Several factors may have contributed to the discrepancies. First, the ongoing ADNI study may have a more heterogeneous MCI group than some of the previously published CSF studies. As pointed out by Hansson et al, ${ }^{50}$ participants included in CSF studies have generally been highly selected, for example, by inclusion of only patients with MCI who progress to AD. In ADNI, the ultimate end point is not known for many patients with MCI. Further, studies have often used dichotomized variables for CSF values and prognosis. ${ }^{50,51}$ A stable/conversion dichotomization involves clinical judgment, which may vary from physician to physician and demands long follow-up intervals impractical for clinical trials. It may be advantageous to identify other preselection criteria, biomarkers, or clinical measures of decline than conversion. Therefore, it is important to relate the biomarkers to easily administered continuous behavioral measures. Most interesting, another study investigating continuous variables $^{52}$ did not find any association between MMSE change and change in CSF levels of either $A \beta 42$, tau, or p-tau $(r=0.18,-.03$, and -.07 , respectively). This does not mean that CSF measures are not related to clinical change. CSF tau/ A $\beta 42$ ratio did correlate in the expected negative direction with change in logical delayed memory in the present sample, but the effect size was too modest to reach significance. Select MR imaging morphometry and FDG-PET measures at baseline were significantly more sensitive to 2-year change in CDR-SB and MMSE than were CSF measures. Both cortical thickness and metabolism of parietal regions of interest served as unique predictors of clinical decline, indicating that even though FDG-PET did not contribute uniquely to diagnostic classification when MR imaging morphometric variance was accounted for, some additional prognostic information can be obtained by combining the 2 imaging modalities.

While the present findings show that the different biomarkers all were sensitive to diagnostic group, a question of great interest is whether the findings regarding specific measures 
can be applied on an individual subject basis. McEvoy et $\mathrm{al}^{7}$ recently reported that semiautomated individually specific quantitative MR imaging methods identical to those used here can be used to identify a pattern of atrophy in MCI that is predictive of conversion to $\mathrm{AD}$ after 1 year. Hence, in light of the present findings also indicating somewhat superior sensitivity of such MR imaging morphometry measures compared with other biomarkers, it does seem that these measures are prime candidates to be used on an individual basis (eg, to enrich clinical trials). However, as seen from Fig 3, while the MR imaging morphometry measures evaluated here do predict 2-year change in screening and memory parameters among patients with MCI, the regression plots also show considerable scatter. Hence, while these measures can yield individual prognostic information, this will be associated with considerable uncertainty, and at present, any such estimate must be made with great caution.

The present results are limited by a number of factors: Participants were selected on the basis of willingness and ability to undergo MR imaging and PET scanning and lumbar puncture and may thus not be fully comparable with other samples. However, imaging is an integral part of the ADNI protocol, so participants did enter with the intention of having brain scans performed, and approximately half of the ADNI participants have also agreed to have CSF samples drawn. ${ }^{53}$ In terms of age, MMSE score, $A \beta 42$, $t$-tau, $p$-tau, and ratios of $t$-tau/A $\beta 42$ and $\mathrm{p}$-tau/A $\beta 42$, the subgroups studied in the present article do appear to be representative of the larger ADNI sample. The mean values for these indices in the present sample appear very similar to those reported by Shaw et $\mathrm{al}^{53}$ for 410 participants with CSF measures, and all the present mean values for age, MMSE, and CSF measures for NC, MCI, and AD deviate less than one-fifth of the SDs from the means reported by Shaw et al for the larger groups. Still, the present sample may, of course, not be fully representative of the general population. Furthermore, the multisite design of the ADNI is likely to add some noise in data collection. Finally, the ADNI study is still ongoing, and the ultimate status of the current MCI group is unknown. That being said, the present study involving multiple sites and 2 years of follow-up likely represents a more realistic model for current clinical trial designs than longer interval single-site studies.

\section{Conclusions}

Each of the biomarkers demonstrated potential to inform diagnosis and/or prognosis and enrich clinical trials. As a single classifier, MR imaging morphometry (hippocampal volume) was the most sensitive to diagnostic group, but the inclusion of CSF biomarkers ( $\mathrm{t}$-tau/A $\beta 42)$ did result in significant improvement of classification (NC/AD). Still, both quantitative MR imaging morphometry and regional metabolism as assessed by coregistered FDG-PET data provided better prediction of clinical decline than did CSF biomarkers. MR imaging morphometry showed somewhat superior diagnostic and prognostic sensitivity and is the least invasive, least expensive, and most widely available method. MR images are often routinely required as part of the diagnostic work-up, so a broader application of MR imaging morphometry may be feasible and useful.

\section{References}

1. Mosconi L, Brys M, Glodzik-Sobanska L, et al. Early detection of Alzheimer's disease using neuroimaging. Exp Gerontol 2007;42:129-38

2. Craig-Schapiro R, Fagan AM, Holtzman DM. Biomarkers of Alzheimer's disease. Neurobiol Dis 2009;35:128 - 40. Epub 2008 Oct 28

3. Blennow K, Wallin A, Agren $\mathrm{H}$, et al. Tau protein in cerebrospinal fluid: a biochemical marker for axonal degeneration in Alzheimer disease? Mol Chem Neuropathol 1995;26:231-45

4. Andreasen N, Blennow K. Beta-amyloid (Abeta) protein in cerebrospinal fluid as a biomarker for Alzheimer's disease. Peptides 2002;23:1205-14

5. Fennema-Notestine C, Hagler DJ Jr, McEvoy LK, et al. Structural MRI biomarkers for preclinical and mild Alzheimer's disease. Hum Brain Mapp 2009;30:3238-53

6. Leow AD, Yanovsky I, Parikshak N, et al. Alzheimer's disease neuroimaging initiative: a one-year follow up study using tensor-based morphometry correlating degenerative rates, biomarkers and cognition. Neuroimage 2009;45: 645-55

7. McEvoy LK, Fennema-Notestine C, Roddey JC, et al. Alzheimer disease: quantitative structural neuroimaging for detection and prediction of clinical and structural changes in mild cognitive impairment. Radiology 2009;251:195-205

8. Misra C, Fan Y, Davatzikos C. Baseline and longitudinal patterns of brain atrophy in MCI patients, and their use in prediction of short-term conversion to AD: results from ADNI. Neuroimage 2009;44:1415-22

9. Mosconi L, Tsui WH, Herholz K, et al. Multicenter standardized 18F-FDG PET diagnosis of mild cognitive impairment, Alzheimer's disease, and other dementias. J Nucl Med 2008;49:390-98

10. de Leon MJ, Ferris SH, George AE, et al. Computed tomography and positron emission transaxial tomography evaluations of normal aging and Alzheimer's disease. J Cereb Blood Flow Metab 1983;3:391-94

11. Langbaum JBS, Chen K, Lee W, et al. Categorical and correlational analyses of baseline fluorodeoxyglucose positron emission tomography images from the Alzheimer's Disease Neuroimaging Initiative. Neuroimage 2009;45:1107-16. Epub 2009 Jan 21

12. De Santi S, de Leon MJ, Rusinek H, et al. Hippocampal formation glucose metabolism and volume losses in MCI and AD. Neurobiol Aging 2001;22: 529-39

13. Mosconi L, Sorbi S, de Leon MJ, et al. Hypometabolism exceeds atrophy in presymptomatic early-onset familial Alzheimer's disease. $\mathrm{J} \mathrm{Nucl} \mathrm{Med}$ 2006;47:1778-86

14. Jagust WJ, Eberling JL, Wu CC, et al. Brain function and cognition in a community sample of elderly Latinos. Neurology 2002;59:378-83

15. Wu CC, Mungas D, Petkov CI, et al. Brain structure and cognition in a community sample of elderly Latinos. Neurology 2002;59:383-91

16. Walhovd KB, Fjell AM, Dale AM, et al. Multi-modal imaging predicts memory performance in normal aging and cognitive decline. Neurobiol Aging 2009. In press

17. de Leon MJ, Mosconi L, Blennow K, et al. Imaging and CSF studies in the preclinical diagnosis of Alzheimer's disease. Ann N Y Acad Sci 2007;1097: $114-45$

18. de Leon MJ, DeSanti S, Zinkowski R, et al. Longitudinal CSF and MRI biomarkers improve the diagnosis of mild cognitive impairment. Neurobiol Aging 2006;27:394-401

19. Chou YY, Lepore N, Avedissian C, et al. Mapping correlations between ventricular expansion and CSF amyloid and tau biomarkers in 240 subjects with Alzheimer's disease, mild cognitive impairment, and elderly controls. Neuroimage 2009;46:394-410. Epub 2009 Feb 21

20. Leow AD, Yanovsky I, Parikshak N, et al. Alzheimer's Disease Neuroimaging Initiative: a one-year follow up study using tensor-based morphometry correlating degenerative rates, biomarkers and cognition. Neuroimage 209;45:645-55

21. Schuff N, Woerner N, Boreta L, et al. MRI of hippocampal volume loss in early Alzheimer's disease in relation to ApoE genotype and biomarkers. Brain 2009; 132(Pt 4):1067-77. Epub 2009 Feb 27

22. Fjell AM, Walhovd KB, Amlien I, et al. Morphometric changes in the episodic memory network and tau pathologic features correlate with memory performance in patients with mild cognitive impairment. AJNR Am J Neuroradiol 2008;29:1183-89

23. Folstein MF, Folstein SE, McHugh PR. "Mini-mental state": a practical method for grading the cognitive state of patients for the clinician. J Psychiatr Res 1975; 12:189-98

24. Wechsler, D. Manual for the Wechsler Memory Scale-Revised. The Psychological Corporation, San Antonio; 1987

25. McKhann G, Drachman D, Folstein M, et al. Clinical diagnosis of Alzheimer's disease: report of the NINCDS-ADRDA Work Group under the auspices of Department of Health and Human Services Task Force on Alzheimer's Disease. Neurology 34:939-44

26. Jovicich J, Czanner S, Greve D, et al. Reliability in multi-site structural MRI studies: effects of gradient non-linearity correction on phantom and human data. Neuroimage 2006;30:436-43

27. Sled JG, Zijdenbos AP, Evans AC. A nonparametric method for automatic 
correction of intensity nonuniformity in MRI data. IEEE Trans Med Imaging 1998;17:87-97

28. Fischl B, Salat DH, Busa E, et al. Whole brain segmentation: automated labeling of neuroanatomical structures in the human brain. Neuron 2002;33: 341-55

29. Makris N, Meyer JW, Bates JF, et al. MRI-based topographic parcellation of human cerebral white matter and nuclei. II. Rationale and applications with systematics of cerebral connectivity. Neuroimage 1999;9:18-45

30. Fischl B, van der Kouwe A, Destrieux C, et al. Automatically parcellating the human cerebral cortex. Cereb Cortex 2004;14:11-22

31. Dale AM, Sereno MI. Improved localization of cortical activity by combining EEG and MEG with MRI cortical surface reconstruction: a linear approach. $J$ Cognitive Neurosci 1993;5:162-76

32. Dale AM, Fischl B, Sereno MI. Cortical surface-based analysis. I. Segmentation and surface reconstruction. Neuroimage 1999;9:179-94

33. Fischl B, Dale AM. Measuring the thickness of the human cerebral cortex from magnetic resonance images. Proc Natl Acad Sci U S A 2000;97:11050-55

34. Fischl B, Sereno MI, Dale AM. Cortical surface-based analysis. II: Inflation, flattening, and a surface-based coordinate system. Neuroimage 1999;9: 195-207

35. Fischl B, Sereno MI, Tootell RB, et al. High-resolution intersubject averaging and a coordinate system for the cortical surface. Hum Brain Mapp 1999;8: 272-84

36. Salat DH, Buckner RL, Snyder AZ, et al. Thinning of the cerebral cortex in aging. Cereb Cortex 2004;14:721-30

37. Rosas HD, Liu AK, Hersch S, et al. Regional and progressive thinning of the cortical ribbon in Huntington's disease. Neurology 2002;58:695-701

38. Kuperberg GR, Broome MR, McGuire PK, et al. Regionally localized thinning of the cerebral cortex in schizophrenia. Arch Gen Psychiatry 2003;60:878-88

39. Desikan RS, Segonne F, Fischl B, et al. An automated labeling system for subdividing the human cerebral cortex on MRI scans into gyral based regions of interest. Neuroimage 2006;31:968-80

40. Walhovd KB, Fjell AM, Amlien I, et al. Multimodal imaging in mild cognitive impairment: metabolism, morphometry and diffusion of the temporal-parietal memory network. Neuroimage 2009;45:215-23

41. Edison P, Archer HA, Hinz R, et al. Amyloid, hypometabolism, and cognition in Alzheimer disease: an [11C]PIB and [18F]FDG PET study. Neurology 2007; 68:501-08. Epub 2006 Oct 25

42. Villain N, Desgranges B, Viader F, et al. Relationships between hippocampal atrophy, white matter disruption, and gray matter hypometabolism in Alzheimer's disease. J Neurosci 2008;28:6174-81

43. Karas G, Sluimer J, Goekoop R, et al. Amnestic mild cognitive impairment structural MR imaging findings predictive of conversion to Alzheimer disease. AJNR Am J Neuroradiol 2008;29:944-49

44. Devanand DP, Habeck CG, Tabert MH, et al. PET network abnormalities and cognitive decline in patients with mild cognitive impairment. Neuropsychopharmacology 2006;31:1327-34

45. Minoshima S, Frey KA, Foster NL, et al. Preserved pontine glucose metabolism in Alzheimer disease: a reference region for functional brain image (PET) analysis. J Comput Assist Tomogr 1995;19:541-47

46. Shaw LM, Korecka M, Clark CM, et al. Biomarkers of neurodegeneration for diagnosis and monitoring therapeutics. Nat Rev Drug Discov 2007;6:295-303. Epub 2007 Mar 9

47. Morris JC. The Clinical Dementia Rating (CDR): current version and scoring rules. Neurology 1993;43:2412-14

48. Wechsler D. Wechsler Memory Scale-Revised. San Antonio: Psychological Corporation; 1987

49. Hanley JA, McNeil BJ. A method of comparing the areas under receiver operating characteristic curves derived from the same cases. Radiology 1983;148: $839-43$

50. Hansson $\mathrm{O}$, Zetterberg $\mathrm{H}$, Buchhave $\mathrm{P}$, et al. Association between CSF biomarkers and incipient Alzheimer's disease in patients with mild cognitive impairment: a follow-up study. Lancet Neurol 2006;5:228-34

51. Hansson $\mathrm{O}$, Buchhave $\mathrm{P}$, Zetterberg $\mathrm{H}$, et al. Combined rCBF and CSF biomarkers predict progression from mild cognitive impairment to Alzheimer's disease. Neurobiol Aging 2009;30:165-73

52. Sluimer JD, Bouwman $\mathrm{FH}$, Vrenken $\mathrm{H}$, et al. Whole-brain atrophy rate and CSF biomarker levels in MCI and AD: a longitudinal study. Neurobiol Aging 2008 Aug 7. [Epub ahead of print]

53. Shaw LM, Vanderstichele H, Knapik-Czajka M, et al. Cerebrospinal fluid biomarker signature in Alzheimer's disease neuroimaging initiative subjects. Ann Neurol 2009;65:403-13 